\title{
Isolation and Identification of Endophytic Bacteria from the Roots of the Endangered Plant Ostrya rehderiana
}

\author{
Taihe Xiang ${ }^{1,2^{*}}$, Xiaxiu Tong ${ }^{1}$, Keyun Wang ${ }^{1}$ and Leizhen Wang ${ }^{1}$ \\ ${ }^{1}$ College of Life and Environment Sciences, Hangzhou Normal University, Hangzhou 310036, China \\ ${ }^{2}$ Zhejiang Provincial Key Laboratory for Genetic Improvement and Quality Control of Medicinal Plants, Hangzhou 310036, China \\ *For correspondence: xthcn@163.com; xthcn@hznu.edu.cn \\ Received 26 August 2019; Accepted 05 September 2019; Published 08 January 2020
}

\begin{abstract}
Ostrya rehderiana Chun, one of the most endangered species in China mainly because of difficult transplantation, is distributed in Western Tianmu Mountain of the Zhejiang Province in China. There are presently only five plants still remaining in the wild. Since plant endophytes affect the growth and development of the host plant either directly or indirectly, in this study, we adopted the tissue block separation method to isolate 22 endophytic bacteria strains from the roots of $O$. rehderiana, with 10 strains from wild $O$. rehderiana and 12 from $O$. rehderiana plantations. From morphological analysis and $16 \mathrm{~S}$ rDNA sequence analysis, 6 known genera were identified from the 22 strains. Among the isolated strains, eight were Bacillus (the dominant endophytic bacteria in the root of $O$. rehderiana), six were Paenibacillus, three were Agrobacterium, three were Paraburkholderia, one was Rhizobium, and one was Pseudomonas. Both wild and plantation O. rehderiana contained Bacillus, Paenibacillus and Paraburkholderia, which presumably could promote the growth of $O$. rehderiana. Besides, the Rhizobium strain isolated from wild $O$. rehderiana might be beneficial to the growth of $O$. rehderiana, but the Agrobacterium strains from the plantations might impair the growth of $O$. rehderiana. (C) 2020 Friends Science Publishers
\end{abstract}

Keywords: Ostrya rehderiana Chun; Root; Endophyte; Phylogenetic tree; Endangered species

\section{Introduction}

Endophytes, including endophytic fungi and endophytic bacteria, are a group of microorganisms that are inseparable from and closely associated with their host plants (Wani et al. 2015). Although previous studies largely focused on endophytic fungi, endophytic bacteria are also receiving rising interests lately in studying the interaction between plants and microorganisms (Pinski et al. 2019). Many endophytic bacteria have been found to possess various beneficial effects for their host, including nitrogen fixation, growth promotion, disease prevention, and stress resistance (Chihaoui et al. 2015; Tétard-Jones and Edwards 2016; Song et al. 2017; Khare et al. 2018; Enebe and Babalola 2019). However, some endophytic bacteria (accounting for about $10 \%-20 \%$ in total) also appeared to be detrimental to the growth and development of their hosts (He et al. 2004; Ellouze et al. 2014). It was found by Peer et al. (1990) that a significantly higher percentage of Pseudomonads obtained from the endorhizosphere $(30 \%)$ reduced plant growth than those obtained from the root surface (4\%). Jin et al. (1996) reported on some endophytic bacteria in wheat seedlings that could inhibit wheat growth to varying extent. Besides, some endophytes demonstrate pathogenic effects in their host plants. Bending et al. (2002) reported that most Bacillus isolates stimulated growth of $S$. luteus along the $P$. sylvestris root, whereas the isolates of Pseudomonas and Serratia inhibited root colonization, and the Burkholderia and Serratia isolates inhibited the formation of the ectomycorrhiza by $97 \%$ and $41 \%$ respectively. Newman et al. (2003) reported that Xylella fastidiosa could cause Pierce's disease as well as several other major agricultural diseases in grapevine. They observed the movement of $X$. fastidiosa through bordered pits to neighboring vessels and proposed that vessel-to-vessel movement was a key colonization strategy whose failure resulted in vessel plugging and disease.

Ostrya rehderiana Chun (O. rehderiana), a plant of the Betulaceae family in the Ostrya genus, is distributed in the Western Tianmu Mountain of the Zhejiang Province in China. It is one of the rarest plants in China and currently there are only five plants grown in the wild. Difficulty in transplantation is one of the main causes of endangerment since transplanted individuals do not readily develop new roots and thus survive (Guan 2008; Shaw et al. 2014). It remains unknown whether the endophytes in the roots are culpable for the difficulty in root development of transplanted individuals. Therefore, this study adopted the 
root block separation method to isolate and purify endophytic bacteria from the roots of $O$. rehderiana, and combined with morphology studies with molecular biology for the identification and evolution analysis of the collected bacteria. The results lay further foundation for understanding the endangerment of $O$. rehderiana and evaluating the effects of its endophytic bacteria on its growth.

\section{Materials and Methods}

\section{Materials}

Root-containing soil blocks were collected from wild $O$. rehderiana plants at the Tianmu Mountain of Hangzhou and from the artificial plantations. The collected soil blocks were cleaned by washing once with tap water to remove dirt and debris on the surface, once more with dilute dishwashing detergent, and then finally rinsed thoroughly with tap water again. Then, the cleaned blocks were immersed in dilute to 1 times of $\mathrm{NaClO}$ (saturated solution) for $30 \mathrm{~min}$. After washing with sterilized water for three times, cut root blocks into small cubes (3-5 mm in length), and placed in Potato Dextrose Agar (PDA) medium that contained $50 \mathrm{mg} / \mathrm{L}$ streptomycin, $200 \mathrm{mg} / \mathrm{L}$ peeled, diced, and filtered potato infusion, $20 \mathrm{~g} / \mathrm{L}$ dextrose, and $14 \mathrm{~g} / \mathrm{L}$ agar. In each Petri dish were evenly placed 3-5 root blocks of uniform size with their section facing the PDA medium. Two control groups were also tested, one with a medium free of any material but sterilized water to verify proper sterilization for inoculation, and the other with root blocks removed from the Petri dish after rolling quickly in the PDA medium to verify thorough sterilization of the block surface. The test and control groups were both cultured in dark at $28^{\circ} \mathrm{C}$ until plaque grew visible on the section. The plaques were numbered and selectively transferred into new medium (PDA with $50 \mathrm{mg} / \mathrm{L}$ streptomycin) for further purification. Single colonies of endophytic bacteria were obtained after 3 cycles of cultivation. The growth rate, size, color, texture, etc., of the separated colonies were observed for an initial identification with reference to the Chinese Handbook of Fungi (Dong and Cai 2001) and Bergey's Manual of Systematic Bacteriology (Bergey’s Manual Trust 2001-2012).

The DNA of the endophytes was extracted with the Ezup spin column bacterial genomic DNA miniprep kit (Sangon Biotech, Shanghai, China) according to the user manual. The PCR amplification of the DNA was carried out using the following primers: $16 \mathrm{~S}$ rDNA of 27f: AGAGTTTGATCMTGGCTCAG and 1492r: TACGGYTACCTTGTTACGACTT; ITS rDNA upstream: TCCGTAGGTGAACCTGCGG and ITS rDNA downstream: GTCCTCCGCTTATT. The PCR amplification was carried out on a PE 9700 synthesizer (Applied Biosystems, C.A., U.S.A.) in a medium $(20 \mu \mathrm{L})$ containing 2×Taq Master Mix (10 $\mu \mathrm{L}$, Sangon Biotech, Shanghai, China), DNA ( $1 \mu \mathrm{L}, 100 \mathrm{ng} / \mu \mathrm{L})$, primer $(1 \mu \mathrm{L}, 10 \mathrm{pmol} / \mu \mathrm{L})$, and double distilled water $(7 \mu \mathrm{L})$ with 30 cycles as follows: denaturation at $94^{\circ} \mathrm{C}$ for $30 \mathrm{~s}$, annealing at $58^{\circ} \mathrm{C}$ for $30 \mathrm{~s}$, elongation at $72^{\circ} \mathrm{C}$ for $1 \mathrm{~min}$. Pre-denaturation was carried out at $94^{\circ} \mathrm{C}$ for $5 \mathrm{~min}$ and final elongation at $72^{\circ} \mathrm{C}$ for $5 \mathrm{~min}$. The products were electrophoresed in $1.5 \%$ agarose gel for $60 \mathrm{~min}$ at $90 \mathrm{~V}$ and stained with $\mathrm{EB}$ or pictured with a $\mathrm{Bio} / \mathrm{Rad}$ gel imager. The electrophoresed PCR products were purified and linked to pMD18-T vector (Takara Bio Inc., Japan) and transcribed into competent E. coli DH5a. The positive clones were screened in lysogeny broth containing $50 \mathrm{mg} / \mathrm{L}$ ampicillin, and the recombinant plasmids were sequenced by Sangon Biotech (Shanghai, China). The species of the isolated endophytes were determined by homology alignment in Blast (http://www.ncbi.nlm.nih.gov/blast) with known nucleic acid sequences in GenBank. Phylogenetic trees were constructed with MEGA6 (http://www.megasoft.net) based on the neighbor-joining method using default parameters.

\section{Results}

\section{Separation and preliminary identifications}

After the root blocks of $O$. rehderiana, whose surface had been sterilized, were cultured in PDA for 2-3 days, cells began to grow on the section. No cell was grown in the control group, which confirmed that the surface of the root blocks had been completely sterilized and the observed cells on the sections were endophytes from within the roots. A total of 22 strains of endophytic bacteria were obtained eventually and numbered as follows, with the plant source indicated in the parenthesis: TM (wild), TMR01 (plantation), TMR02 (plantation), TMR03 (plantation), TMR04-1 (plantation), TMR04-2 (plantation), TMR05 (plantation), TMR06-1 (plantation), TMR06-2 (plantation), TMW07-1 (wild), TMW07-2 (wild), TMR08 (plantation), TMR09 (plantation), TMR10 (plantation), TMR13 (plantation), TMW14 (wild), TMW15 (wild), TMW16 (wild), TMW17 (wild), TMW19-1 (wild), TMW19-2 (wild), and TMW20 (wild). Among them, 10 strains were from wild plants and 12 strains were from plantations (Fig. 1). The cultivated colonies were deemed to be endophytic bacteria according to their surface morphology, color, texture, growth rate, edge morphology, etc.

\section{Molecular identification and evolution analysis}

All DNA isolated from 22 strains amplification with $16 \mathrm{~S}$ rRNA general primers gave a single band with a size of 900-1500 bp (Fig. 2), whereas no band was obtained when the ITS rDNA general primer was used. After the PCR products were cloned, the recombinant plasmids of the positive clones identified by restriction endonuclease were sequenced, and the resulting sequences were found to highly similar to sequences in GenBank database. Since Blast analysis showed that all strains could be traced to some 

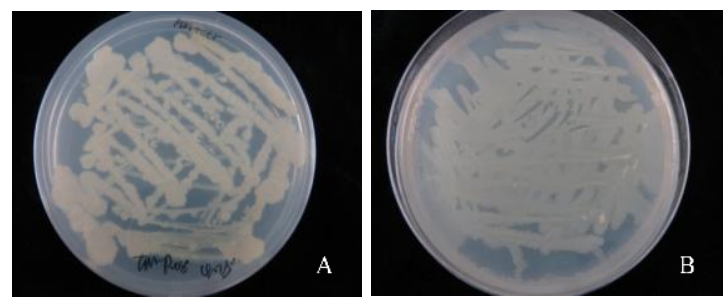

Fig. 1: Endophytic bacteria from the root of Ostrya rehderiana. A: TMR08 strain; B: TMW15 strain

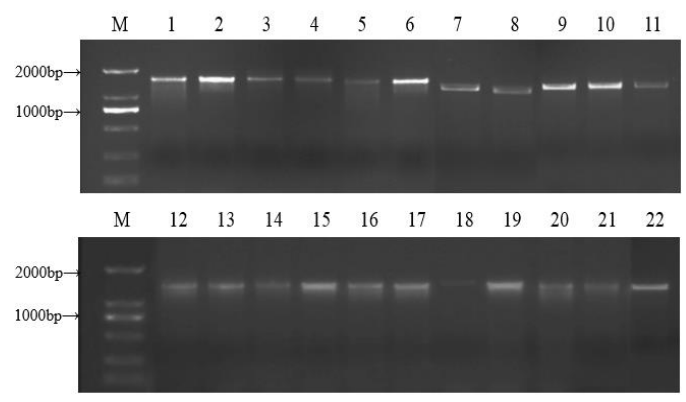

Fig. 2: PCR results for $16 \mathrm{~S}$ rDNA amplifying sequencing of the 22 endophytic bacteria strains. M: DL 2000 DNA marker; the numbers 1-22 stand for the bacteria strains of TM, TMR01, TMR02, TMR03, TMR04-1, TMR04-2, TMR05, TMR06-1, TMR06-2, TMW07-1, TMW07-2, TMR08, TMR09, TMR10, TMR13, TMW14, TMW15, TMW16, TMW17, TMW19-1, TMW19-2, and TMW20, respectively

strain with a high level of homology in GenBank database, the 22 strains were classified into 6 known genera, including 8 strains of Bacillus (TMR01 (GenBank accession: MK615900), TMR02 (GenBank accession: MK615901), TMR04-1 (GenBank accession: MK615902), TMR06-1 (GenBank accession: MN186891), TMR10 (GenBank accession: MK615909), TMW17 (GenBank accession: MK615914), TMW19-1 (GenBank accession: MK61591), and TMW20 (GenBank accession: MK615916)), 6 strains of Paenibacillus (TMR04-2 (GenBank accession: MK615903), TMW07-1 (GenBank accession: MK615905), TMW07-2 (GenBank accession: MK615906), TMW14 (GenBank accession: MK615911), TMW15 (GenBank accession: MK615912), and TMW16 (GenBank accession: MK615913)), 3 strains of Agrobacterium (TMR05 (GenBank accession: MK615904), TMR08 (GenBank accession: MK615907), and TMR09 (GenBank accession: MK615908)), 3 strains of Paraburkholderia (TMR03 (GenBank accession: MN150518), TMR06-2 (GenBank accession: MN150520), and TMW19-2 (GenBank accession: MN150516)), 1 strain of Rhizobium (TM (GenBank accession: MK615899)), 1 strain of Pseudomonas (TMR 13 (GenBank accession: MK615910)). Among them, Bacillus accounted for $36.36 \%$ of the total bacteria and was thus the dominant endophytic bacteria, followed by Paenibacillus, which accounted for $27.27 \%$ of the total. The 16S rDNA sequence analysis of endophytic bacteria in the same genus showed high similarity among TMR01, TMR02, TMR04-1, and TMW17 in Bacillus, high similarity among TMR10, TMW19-1 and TMW20 in Bacillus, high similarity between TMR04-2 and TMW07-1 in Paenibacillus, high similarity among TMW07-2, TMW14, and TMW15 in Paenibacillus, high similarity between TMR05 and TMR08 in Agrobacterium, and high similarity between TMR03 and TMR06-2 in Paraburkholderia. Phylogenetic trees were then constructed with MEGA6 (Fig. 3).

\section{Discussion}

Bacillus protects plants from pathogens and pests because it can compete with harmful pathogens for nutrients and space, synthesize antibacterial metabolites or hydrolase to destroy the cell structure of pathogens, and prevent or reduce diseases caused by pathogens (Lopes et al. 2018). Gao et al. (2015) reported that the endophytic Bacillus subtilis strain E1R-J was a promising biocontrol agent for wheat powdery mildew. Paraburkholderia can promote plant growth and may suppress diseases (Donoso et al. 2016; Zúñiga et al. 2017). Rhizobium can assist nitrogen fixation to provide abundant nitrogen sources for the growth of plant roots. Zhao et al. (2018) colonized the strain Rhizobium sp. IRBG74 into the roots of Arabidopsis thaliana seedlings and noted enhanced formation and growth of lateral roots. Agrobacterium is a Gram-negative bacterium, which has no nitrogen fixation function and mostly causes plant tumors (Zupan and Zambryski, 1995; Barton et al. 2018). Brunner and Pootjes (1969) reported that Agrobacterium tumefaciens V-1 could cause tumors in plants. Pseudomonas is often beneficial to plant growth. Zhou et al. (2016) reported that Pseudomonas fluorescens ALEB7B, an endophytic bacterium isolated from the endangered Chinese medicinal plant Atractylodes lancea, could produce nitrogen-containing volatile substances ingested by the host plant. The volatile substances not only promoted plant growth significantly but also activated the plant defense response and significantly promoted the accumulation of active ingredients in the plant. In this work, 22 strains of endophytic bacteria were isolated from the roots of $O$. rehderiana grown both in the wild and in plantations. Bacillus, Paenibacillus, Paraburkholderia, Rhizobium were isolated from the roots of wild $O$. rehderiana, whereas Bacillus, Paenibacillus, Pseudomonas, Paraburkholderia, and Agrobacterium were isolated from the roots of the plantation. The Rhizobium isolated from the roots of the wild plants was beneficial to plant growth. Both Pseudomonas and Agrobacterium were isolated from the plantation. Although Pseudomonas was beneficial to the colonization and growth of the roots of the artificially transplanted $O$. rehderiana, Agrobacterium could cause plant tumors and might not be beneficial to plant growth. Since Bacillus, Paenibacillus, and Paraburkholderia were 
Endophytic Bacteria from the Endangered Plant O. rehderiana / Intl J Agric Biol, Vol 23, No 1, 2020

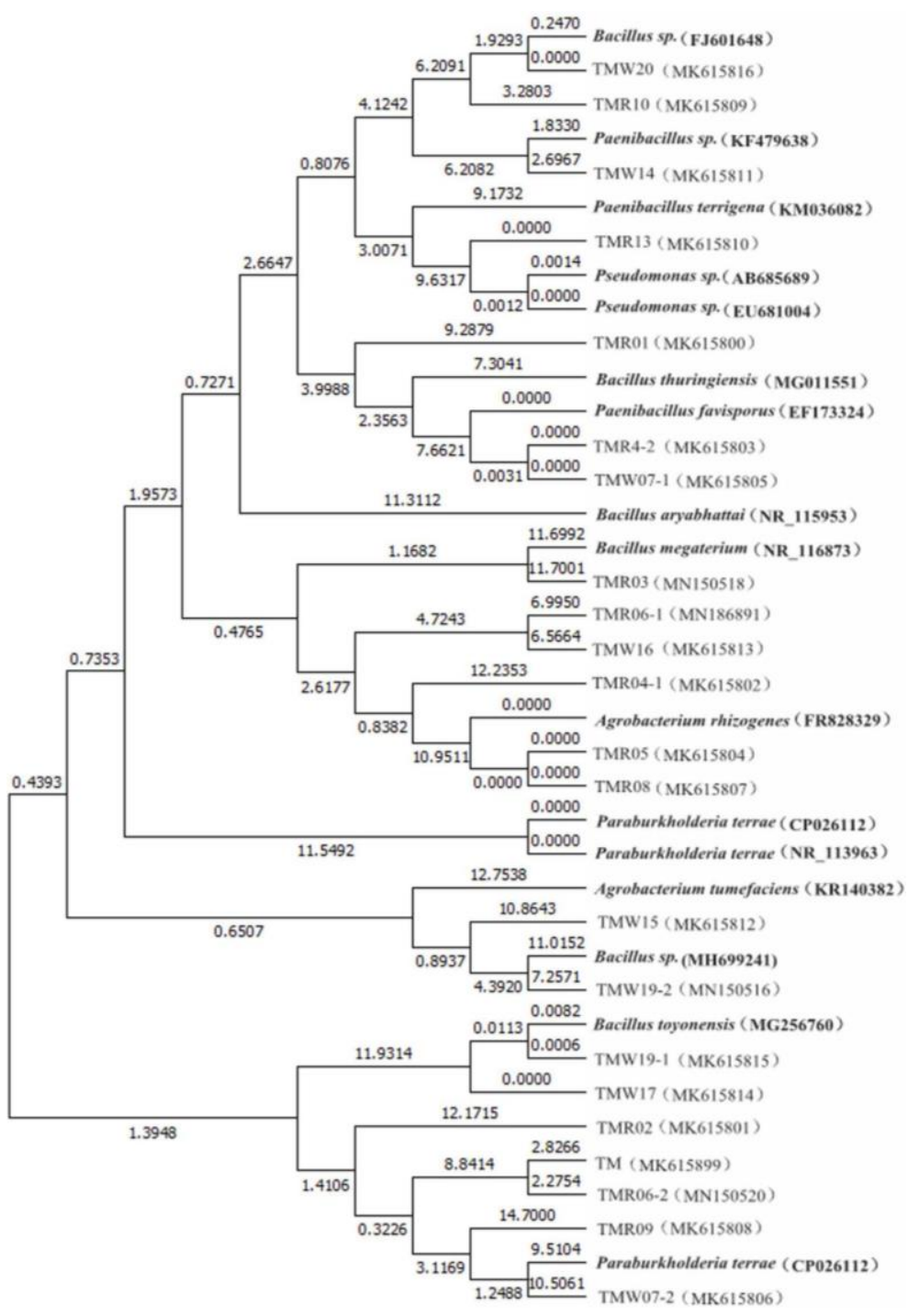

Fig. 3: Phylogenetic trees of the endophytic bacteria based on their 16S rDNA sequences

all found in the wild plants and the plantations, they probably could facilitate the growth of $O$. rehderiana.

\section{Conclusion}

Out of 22 endophytic bacterial strains isolated from the roots of $O$. rehderiana, eight were Bacillus, six were Paenibacillus, three were Paraburkholderia, three were Agrobacterium, one was Rhizobium, and one was Pseudomonas. The results give a clue that some isolated endophytic bacteria strains may be beneficial to plant growth and help to plantation of $O$. rehderiana.

\section{Acknowledgments}

The work was supported by grants from the National Key Research and Development Project of China (Grant No. 2016YFC0503100).

\section{References}

Barton IS, C Fuqua, TG Platt (2018). Ecological and evolutionary dynamics of a model facultative pathogen: Agrobacterium and crown gall disease of plants. Environ Microbiol 20:16-29

Bending GD, EJ Poole, JM Whipps, DJ Read (2002). Characterisation of bacteria from Pinus sylvestris-Suillus luteus mycorrhizas and their effects on root-fungus interactions and plant growth. FEMS Microbiol Ecol 39:219-227

Bergey's Manual Trust, 2001-2012. Bergey's Manual of Systematic Bacteriology, $2^{\text {th }}$ edn, Vol. 1-5. Springer-Verlag, New York, USA

Brunner M, CF Pootjes (1969). Bacteriophage release in a lysogenic strain of Agrobacterium tumefaciens. J Virol 3:181-186

Chihaoui SA, D Trabelsi, A Jdey, H Mhadhbi, R Mhamdi (2015). Mhadhbi and R. Mhamdi. Inoculation of Phaseolus vulgaris with the noduleendophyte Agrobacterium spp. 10C2 affects richness and structure of rhizosphere bacterial communities and enhances nodulation and growth. Arch Microbiol 197:805-813

Dong X, M Cai (2001. Manual of Systematic Identification of Common Bacteria, p: 62. Science Press, Beijing, China (in Chinese) 
Donoso R, P Leiva-Novoa, A Zúñiga, T Timmermann, G RecabarrenGajardo, B González (2016). Biochemical and genetic bases of indole-3-acetic acid (auxin phytohormone) degradation by the plantgrowth-promoting Rhizobacterium Paraburkholderia phytofirmans PsJN. Appl Environ Microbiol 83:1-20

Enebe MC, OO Babalola (2019). The impact of microbes in the orchestration of plants' resistance to biotic stress: a disease management approach. Appl Microbiol Biotechnol 103:9-25

Ellouze W, AE Taheri, LD Bainard, C Yang, N Bazghaleh, A NavarroBorrell, K Hanson, C Hamel (2014). Soil fungal resources in annual cropping systems and their potential for management. Biomed Res Intl 2014:1-16

Gao X, Y Gong, Y Huo, Q Han, Z Kang, L Huang (2015). Endophytic Bacillus subtilis strain E1R-J is a promising biocontrol agent for wheat powdery mildew. Biomed Res Intl 2015:1-9

Guan S (2008). A Study on the Conservation Genetics of Ostrya Rehderiana Chun, Dissertation. East China Normal University, Shanghai, China

He H, S Qiu, F Hu, X Guan (2004). Advance in biological effects of endophytic bacteria. J Microbiol 24:40-45

Jin L, F Ba, P Ji, R Mei (1996). Discovery of deleterious endobacteria and their effects on growth of wheat. Acta Phytopathol Sin 26:123-126

Khare E, J Mishra, NK Arora (2018). Multifaceted interactions between endophytes and plant: developments and prospects. Front Microbiol 9:1-12

Lopes R, S Tsui, PJRO Gonçalves, MVD Queiroz (2018). A look into a multifunctional toolbox: endophytic Bacillus species provide broad and underexploited benefits for plants. World J Microbiol Biotechnol 34:94

Newman KL, RPP Almeida, AH Purcell, SE (2003). Lindow. Use of a green fluorescent strain for analysis of Xylella fastidiosa colonization of Vitis vinifera. Appl Environ Microbiol 69:7319-7327
Peer RV, HL Punte, LAD Weger, B Schippers (1990). Characterization of root surface and endorhizosphere Pseudomonads in relation to their colonization of roots. Appl Environ Microbiol 56:2462-2470

Pinski A, A Betekhtin, K Hupert-Kocurek, LAJ Mur, R Hasterok (2019). Defining the genetic basis of plant-endophytic bacteria interactions. Intl J Mol Sci 20:1-32

Shaw K, L Stritch, S Roy, B Wilson, R Govaerts (2014). Ostrya rehderiana The IUCN Red List of Threatened Species. 2014e.T32304A2813136

Song Y, P Wu, Y Li, X Tong, Y Zheng, Z Chen, L Wang, T Xiang (2017). Effect of endophytic fungi on the host plant growth, expression of expansin gene and flavonoid content in Tetrastigma hemsleyanum Diels \& Gilg ex Diels. Plant Soil 417:393-402

Tétard-Jones C, R Edwards (2016). Potential roles for microbial endophytes in herbicide tolerance in plants. Pest Manage Sci 72:203-209

Wani ZA, N Ashraf, T Mohiuddin, S Riyaz-Ul-Hassan (2015). Plantendophyte symbiosis, an ecological perspective. Appl Microbiol Biotechnol 99:2955-2965

Zhao CZ, J Huang, P Gyaneshwar, D Zhao (2018). Rhizobium spp. IRBG74 alters Arabidopsis root development by affecting auxin signaling. Front Microbiol 8:1-12

Zhou JY, X Li, JY Zheng, CC Dai (2016). Volatiles released by endophytic Pseudomonas fluorescens promoting the growth and volatile oil accumulation in Atractylodes lancea. Plant Physiol Biochem 101:132-140

Zúñiga A, RA Donoso, D Ruiz, GA Ruz, B González (2017). Quorumsensing systems in the plant growth-promoting bacterium Paraburkholderia phytofirmans PsJN exhibit cross-regulation and are involved in biofilm formation. Mol Plant Microbe Interact 30:557-565

Zupan JR, P Zambryski (1995). Transfer of T-DNA from Agrobacterium to the plant cell. Plant Physiol 107:1041-1047 